\title{
Detection of Boundary Defects using Machine Vision and Image Processing
}

\author{
V. S. Jadhav ${ }^{1}$, Dr. D. S. Watvisave ${ }^{2}$ \\ Student, Department of Mechanical (design) Engineering, S.C.O.E, Pune, India ${ }^{1}$ \\ Asst. Professor, Department of Mechanical Engineering, S.C.O.E, Pune, India ${ }^{2}$
}

\begin{abstract}
Machine Vision Systems find their major applications in the industrial sector. But their use in the field of detection of boundary defects and analysis have been realized which is the reason why extensive research is being carried out in this direction. The current work is an attempt to utilize image processing technique in the direction of detection of defects through Machine Vision systems. For this, various components necessary for a simple Machine Vision System will be assembled. A photoelastic spur gear specimen will be taken for study and image processing will be performed on the acquired image using a DSLR camera. Boundary design and defects will be studied by binarization of the image and interpretation of the acquired data in a computer language called Python. The work is carried out in the direction of successfully incorporating this technique of defects detection in future Machine vision systems.
\end{abstract}

Keywords: Boundary defects, Photoelasticity, Machine vision, Image processing.

\section{INTRODUCTION}

Vision technology is a young discipline which deals with images or sequences of images with the objective of manipulating and analysing them in order to look for any deviation from the normal state of existence of a particular surface. Machine Vision refers the industrial application of Vision technology which is a interdisciplinary technology that combines lighting, optics, electronics, information technology, software and automation technology. These are employed in the early production stages where defective parts are immediately removed from the manufacturing process and not finished. This is combined with Photoelasticity which deals with experimental techniques that use properties of light propagating through loaded or deformed components to determine and analyse the relative displacements in the material and state of strains and stresses. It involves models which are constructed from materials that are transparent to the light being used when viewed under a specific instrumentation called as a Polariscope.

The incorporation of Machine Vision system in the direction of specimen boundary detection and analysis using Photoelasticity is the aim of the present work. In such a system, the need for systematic detection and inspection of defects have been realised in different processes. Machine vision is a subcategory of engineering machinery which deals with issues of visual inspection, optics and mechanics in industrial automation. In the present work, a Machine vision system which is used to detect boundary defects on a photoelastic material is constructed and explained.

The whole experiment is studied with the key steps involved in a machine vision system i.e. Image acquisition, Image segmentation and analysis. It is carried out in order to obtain details of a acquired image by image processing so that it irregularities in structures can be found out.

\section{EXPERIMENTAL PROCEDURE}

A. Image Acquisition

The first step consists of recording the data in the form of a digital image so that further analysis can be carried out. Here, first a conventional photoelasticity experiment is carried out in which a photoelastic involute spur gear is loaded in the circular Polariscope. The specimen details are as follows -

Material of the gear - Araldite CY230 with hardener HY951

Number of teeth -23

Pitch diameter $-22.5 \mathrm{~mm}$

Addendum - $2.5 \mathrm{~mm}$

Dedendum $-2 \mathrm{~mm}$

Root circle diameter $-20.5 \mathrm{~mm}$

Face width $-8 \mathrm{~mm}$

Working depth $-4 \mathrm{~mm}$

The basic components of a vision system consist of a frame grabber, a digitizer, light source to illuminate the specimen, a Polariscope, and a processor. The setup is as shown in figure 1.

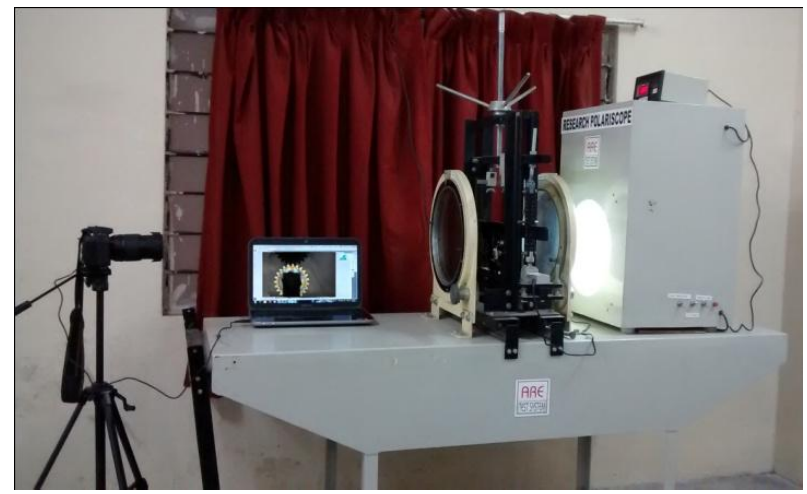

Fig. 1. A simple Machine Vision System.

The image to be analysed is captured with a digital image acquisition device. The input device used for this purpose is a digital camera. The specimen or object to be studied is 
illuminated with a light source. The position of this light source is decided according to the position of the frame grabber and the type of Machine vision system in operation. A digitalize device and convert image from a digital camera to digital format (usually up to twodimensional array whose elements refer to the individual image pixels). The image in digital form is saved to computer memory, for its subsequent processing by the machine vision software or any compatible image processing software. Thus, image sensing and image digitization are performed in the digital input device itself after the frame grabber has performed its operation. The resultant image is shown in the figure 2.

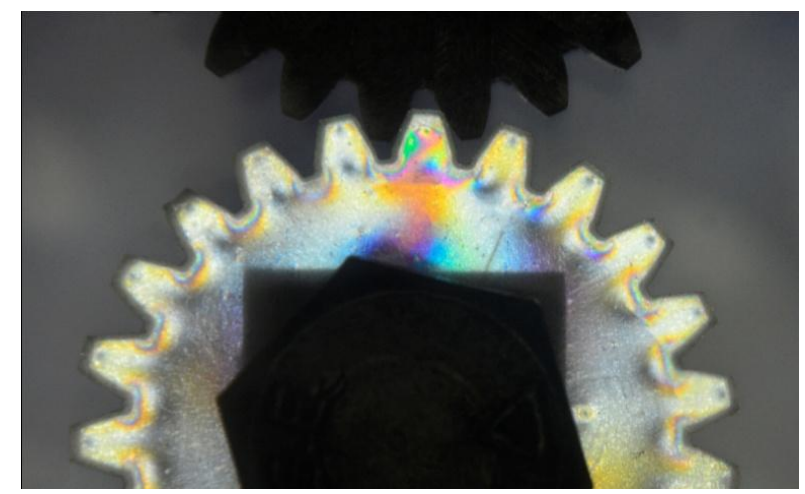

Fig. 2. Acquired Image

\section{B. Image Segmentation}

The next step right after the image is acquired and saved in a processor is Image segmentation. This comprises of converting the image into a binary image i.e. consisting of mostly two colours such as a black and white image. This can be done by thresholding the image. When it is thresholded, image shows just two colours i.e. black and white. This helps in separating the object to be analysed from the other part of the image. For example, its background can be separated from the object in the image. Objects may be uniformly darker or brighter than the background against which they appear. This helps to know which groups of pixels in a digital image correspond to the objects to be analysed. Figure 3 shows the thresholded image of the actual acquired image.

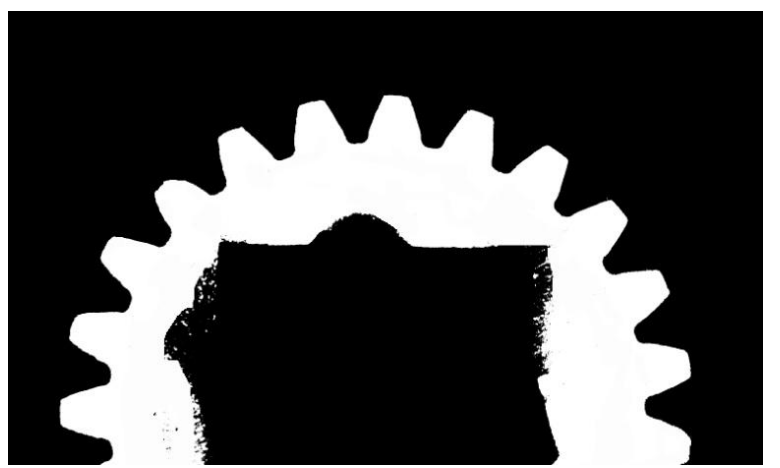

Fig. 3. Acquired image after Thresholding

Ideally, for a binary image like a threshold image, the pixels observed would be black i.e. 0 intensity and white having maximum intensity. But if the objects and background occupy different ranges of gray levels, we can mark the object pixels. This process is called as Thresholding. This image processing technique can be used to separate the background from the boundary of the desired specimen and once it is achieved, it helps in finding the irregularities in a structure out of its regular form.

\section{THE ANALYSIS PROCEDURE}

In the procedure, after the image is segmented by converting it into a binary threshold image, defects in boundary can be detected by help of a computer programme language known as python. It detects the irregularities in the original form of the object by determining the binary data on the region of interest.

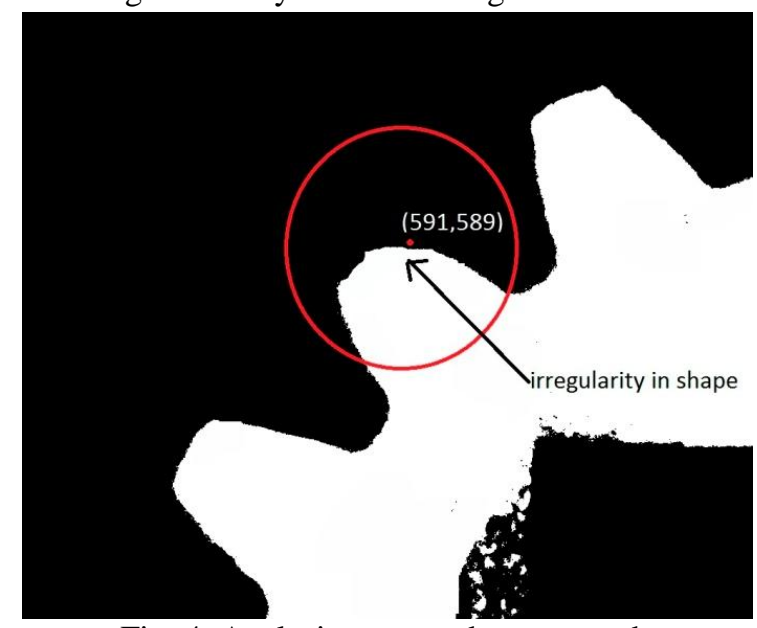

Fig. 4. Analysis area on the gear tooth

In the acquired image of photoelastic involute spur gear, the marked gear tooth will be tested for irregularities. Here, the region of the tooth will be checked for binary data (black or white intensity). The design dimensions are fed into the machine vision system and it is checked that all the specimen boundaries show uniform binary output (in this case, maximum intensity data as white) within specified dimensions. Python selects and scans the whole area by going by co-ordinates on the threshold image and gives result in RGB intensities. Whites represent maximum intensity $(255,255,255)$ and black represents minimum intensity $(0,0,0)$. The code is written in python as shown in the figure 5 to obtain the RGB values at the co-ordinates near the boundary of the gear teeth to see whether they are within the specified dimensions.

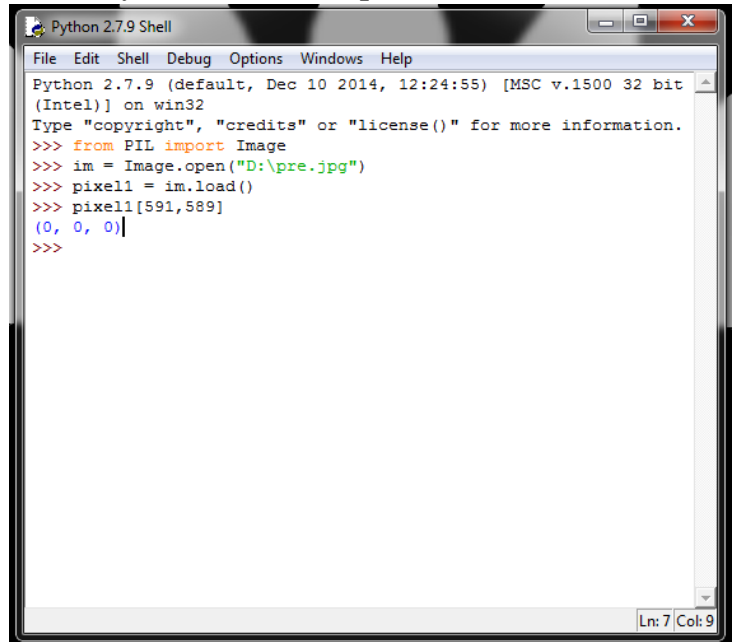

Fig. 5. Result output in Python. 
The results show $(0,0,0)$ RGB intensities at the selected co-ordinates on the image. This shows the presence of irregularities in the form of the gear tooth as it was expected to be a maximum intensity $(255,255,255)$ area according to the original design dimensions.

\section{IV.CONCLUSION}

The current work is based on studying experimental procedure using Machine Vision system to detect and analyse boundary defects on an involute spur gear specimen by application of three key procedures which involves Image Acquisition, Image Segmentation and Image Analysis. The technique proves to be an important component of a Machine vision system in detecting the boundary defects by simple process of binarization. The significance of this technique lies in the fact that simple cameras can be used to acquire images and known simple computer programmes can carry out the analysis. All these components, if together packaged into single software can prove to be a great Machine vision system for detecting boundary defects on any possible geometry.

\section{ACKNOWLEDGMENT}

The authors wish to acknowledge S.C.O.E, Pune and other concerned contributors for the co-operation and support in carrying out the current work.

\section{REFERENCES}

[1] M.N.Buradkar, Dr. D.V. Bhope, S. D. Khamankar, Experimental and photoelastic analysis, international journal of advanced engineering research and studies, June 2012.

[2] Remigiusz Labudzki, Stanislaw Legutko, Applications of Machine Vision, Poznan University of Technology, Faculty of Mechanical Engineering and Management, Poland, August 2012, Vol 1.

[3] Claus-E. Liedtke, New machine vision applications in germany, Institut für Theoretische Nachrichtentechnik und Informationsverarbeitung Universität Hannover, Germany, March 2011, Vol 2, Pp. 1-11.

[4] W. T. Moody, H. B. Phillips, Photoelastic and Experimental analog procedures, Technical and Foreign Services Branch Denver Federal Center Denver, Colorado, March 2000, Vol 3, Pp.1-10.

[5] Larry Davis, machine vision systems, July 2010, Vol. 1, Pp. 1-12. 